\title{
LINEAR OPTIMIZATION WITH MULTIPLE EQUITABLE CRITERIA (*)
}

\author{
by Michael M. Kostreva $\left({ }^{1}\right)$ and Włodzimierz OGryczak $\left({ }^{2}\right)$
}

Communicated by Franco GIANNESSI

\begin{abstract}
The standard multiple criteria optimization starts with an assumption that the criteria are incomparable. However, there are many applications in which the criteria express ideas of allocation of resources meant to achieve some equitable distribution. This paper focuses on solving linear multiple criteria optimization problems with uniform criteria treated in an equitable way. An axiomatic definition of equitable efficiency is introduced as an refinement of Pareto-optimality. Various generation techniques are examined and the structure of the equitably efficient set is analyzed.
\end{abstract}

Keywords: Multiple criteria, linear programming, efficiency, equity.

Résumé. - L'optimisation multicritère classique débute avec l'hypothèse que les critères ne sont pas comparables. Il y a cependant beaucoup d'applications où les critères expriment des idées d'affectation de ressources en vue de quelque répartition équitable. Notre article est concentré sur la résolution de problèmes d'optimisation multicritère avec des critères uniformes traités d'une façon équitable. Nous introduisons une définition de l'efficacité équitable comme un affinement de l'optimalité à la Pareto. Nous examinons diverses techniques de génération et analysons la structure de l'ensemble des points équitablement efficaces.

Mots clés : Critères multiples, programmation linéaire, efficacité, équité.

\section{INTRODUCTION}

The problem of multiple criteria optimization has been studied for many years, and the models of multiple criteria optimization have found their way into many diverse applications [1-3]. The standard approach starts with an assumption that the criteria are incomparable, i.e. having no basis of comparison. However, there are many applications in which the criteria express ideas of allocation of resources and try to achieve some equitable allocation of resources. The main focus of the current paper is solving this foundational problem for the case of linear multiple criteria optimization.

(*) Received December 1996.

( $\left.{ }^{1}\right)$ Clemson University, Mathematical Sciences, Clemson, South Carolina, SC 29634, U.S.A.

(2) Warsaw University, Mathematics \& Computer Science, 02-097 Warsaw, Poland. 
We are motivated by general resource allocation models [4], which have a natural requirement for equitable outcomes. Such problems, prevalent among problems of allocation of resources with respect to many criteria have not been handled in a satisfactory way in the literature. Typically, only minimax (maximin) approaches are studied [5-7] which ignore the rich mathematical structure of a variety of equitable solutions. Another class of applications, arise in location theory, in which the clients of a system are entitled to equitable treatment according to community regulations. In such problems, the decisions often concern the placement of a service center or other facility in a position so that the users are treated in an equitable way, relative to certain criteria [8]. Another type of model is that of approximation of discrete data by a functional form. The residuals may be viewed as objectives to be minimized, and in the classical approach, there is no reason to treat them in any way but equitably.

Indeed, in approaches which seek to scalarize the multiple criteria, some effort is always placed in achieving some comparable functions (pseudocriteria) which are combined to form a final scalar objective function to be optimized. This is done in order to make the physical units of the pseudo-criteria uniform, so that they can be added or otherwise composed. This phase of the modeling discipline seldom questions the process or the consequences of such a uniformization.

One of the results of our research is to trace the consequences of this uniformization beyond the process of aggregation of functions for scalarization. We will show that there are many logical consequences of the assumption, or the provision, that all criteria are equitable.

Consider a decision problem defined as a linear optimization problem with $m$ uniform objective functions $f_{i}(\mathbf{x})=\mathbf{c}^{i} \mathbf{x}$. For simplification we assume, without loss of generality, that the objective functions are to be minimized. The problem can be formulated as follows

$$
\min \{\mathbf{C x}: \mathbf{x} \in Q\}
$$

where

$\mathbf{C}$ is an $m \times n$ matrix (consisting of rows $\mathbf{c}^{i}$ ) representing the vectorfunction that maps the decision space $X=R^{n}$ into the criterion space $Y=R^{m}$,

$Q \subset X$ denotes the feasible set defined by a system of linear equations and inequalities,

$\mathbf{x} \in X$ denotes the vector of decision variables. 
We refer to the elements of the criterion space as achievement vectors. An achievement vector $\mathbf{y} \in Y$ is attainable if it expresses outcomes of a feasible solution $\mathbf{x} \in Q(\mathbf{y}=\mathbf{C x})$. The set of all the attainable achievement vectors is denoted by $A$, i.e. $A=\{\mathbf{y}=\mathbf{C x}$ : for some $\mathbf{x} \in Q\}$.

Model (1) only specifies that we are interested in minimization of all objective functions $f_{i}$ for $i \in I=\{1,2, \ldots, m\}$. In order to make it operational, one needs to assume some solution concept specifying what it means to minimize multiple objective functions. The solution concepts are defined by properties of the corresponding preference model. We assume that solution concepts depend only on evaluation of the achievement vectors while not taking into account any other solution properties not represented within the achievement vectors. Thus, we can limit our considerations to the preference model in the criterion space $Y$.

The preference model is completely characterized by the relation of weak preference [9], denoted hereafter with $\preceq$. Namely, the corresponding relations of strict preference $\prec$ and indifference $\cong$ are defined by the following formulas

$$
\begin{gathered}
\mathbf{y}^{\prime} \prec \mathbf{y}^{\prime \prime} \Leftrightarrow\left(\mathbf{y}^{\prime} \preceq \mathbf{y}^{\prime \prime} \text { and not } \mathbf{y}^{\prime \prime} \preceq \mathbf{y}^{\prime}\right) \\
\mathbf{y}^{\prime} \cong \mathbf{y}^{\prime \prime} \Leftrightarrow\left(\mathbf{y}^{\prime} \preceq \mathbf{y}^{\prime \prime} \text { and } \mathbf{y}^{\prime \prime} \preceq \mathbf{y}^{\prime}\right) .
\end{gathered}
$$

The preference model related to the standard Pareto-optimal solution concept also assumes that the preference relation $\preceq$ is reflexive.

$$
\mathbf{y} \preceq \mathbf{y}
$$

transitive

$$
\left(\mathbf{y}^{\prime} \preceq \mathbf{y}^{\prime \prime} \text { and } \mathbf{y}^{\prime \prime} \preceq \mathbf{y}^{\prime \prime \prime}\right) \Rightarrow \mathbf{y}^{\prime} \preceq \mathbf{y}^{\prime \prime \prime}
$$

and strictly monotonic

$$
\mathbf{y}-\varepsilon \mathbf{e}_{i} \prec \mathbf{y} \quad \text { for } \quad \varepsilon>0
$$

where $\mathbf{e}_{i}$ denotes the $i$-th unit vector in the criterion space. The last assumption expresses the fact that for each individual objective function less is better (minimization). The preference relations satisfying axioms (4-6) are called hereafter rational preference relations. The rational preference relations allow us to formalize the Pareto-optimal solution concept with the following definitions.

vol. 33, n 3, 1999 
DefinITION 1.1: We say that achievement vector $\mathbf{y}^{\prime} \in Y$ rationally dominates $\mathbf{y}^{\prime \prime} \in Y$, or $\mathbf{y}^{\prime \prime}$ is rationally dominated by $\mathbf{y}^{\prime}$, iff $\mathbf{y}^{\prime} \prec \mathbf{y}^{\prime \prime}$ for all rational preference relations $\preceq$.

Definition 1.2: We say that achievement vector $\mathbf{y} \in A$ is rationally nondominated, iff there does not exist $\mathbf{y}^{\prime} \in A$ such that $\mathbf{y}^{\prime}$ rationally dominates $\mathbf{y}$.

DefinItion 1.3: We say that feasible solution $\mathrm{x} \in Q$ is an efficient or Pareto-optimal solution of the multiple criteria problem (1), iff $\mathbf{y}=\mathbf{f}(\mathbf{x})$ is rationally nondominated.

Similar to the relation of rational dominance, we can define the relation of rational indifference (indifference for all rational preference relations) and the relation of rational weak dominance (weak preference for all rational preference relations). The relations of rational dominance $\prec_{r}$, indifference $\widetilde{=}_{r}$ and weak dominance $\prec_{r}$ satisfy conditions (2-3). So, they define the preference relation $\preceq_{r}$. Moreover, relation $\preceq_{r}$ has properties of reflexivity (4), transitivity (5) and strict monotonicity (6), thus it is a rational preference relation. Relation $\prec_{r}$ is the most general rational preference relation and each rational preference relation $\preceq$ is consistent with it in the sense that

$$
\mathbf{y}^{\prime} \preceq_{r} \mathbf{y}^{\prime \prime} \Rightarrow \mathbf{y}^{\prime} \preceq \mathbf{y}^{\prime \prime}
$$

Therefore, an achievement vector $\mathbf{y}^{0} \in A$ is rationally nondominated, if and only if, there exists a rational preference relation $\preceq$ such that $\mathbf{y} \nprec \mathbf{y}^{0}$ for any $\mathbf{y} \in A$.

The rational dominance relation $\preceq_{r}$ may be expressed in terms of vector inequality $\leqq$, where

$$
\begin{aligned}
& \mathbf{y}^{\prime} \leqq \mathbf{y}^{\prime \prime} \Leftrightarrow \mathbf{y}_{i}^{\prime} \leq \mathbf{y}_{i}^{\prime \prime} \quad \text { for } \quad i=1,2, \ldots, m \\
& \mathbf{y}^{\prime} \leq \mathbf{y}^{\prime \prime} \Leftrightarrow\left(\mathbf{y}^{\prime} \leqq \mathbf{y}^{\prime \prime} \text { and not } \mathbf{y}^{\prime \prime} \leqq \mathbf{y}^{\prime}\right) \\
& \mathbf{y}^{\prime}=\mathbf{y}^{\prime \prime} \Leftrightarrow\left(\mathbf{y}^{\prime} \leqq \mathbf{y}^{\prime \prime} \text { and } \mathbf{y}^{\prime \prime} \leqq \mathbf{y}^{\prime}\right)
\end{aligned}
$$

Proposition 1.1: For any achievement vectors $\mathbf{y}^{\prime}, \mathbf{y}^{\prime \prime} \in Y$

$$
\begin{gathered}
\mathbf{y}^{\prime} \preceq_{r} \mathbf{y}^{\prime \prime} \Leftrightarrow \mathbf{y}^{\prime} \leqq \mathbf{y}^{\prime \prime} \\
\mathbf{y}^{\prime} \prec_{r} \mathbf{y}^{\prime \prime} \Leftrightarrow \mathbf{y}^{\prime} \leq \mathbf{y}^{\prime \prime} .
\end{gathered}
$$


As a consequence of Proposition 1.1 we can state that a feasible solution $\mathbf{x}^{0} \in Q$ is an efficient solution of the multiple criteria problem (1), if and only if, there does not exist $\mathbf{x} \in Q$ such that $\mathbf{C x} \leq \mathbf{C x}^{0}$. The latter refers to the commonly used definition of the efficient solutions as feasible solutions for which one cannot improve any criterion without worsening another [1]. However, the axiomatic definition of the rational preference relation allows us to introduce additional properties of the preferences related to the uniform criteria and their equity.

While dealing with uniform criteria, we assume that the preference model is impartial (anonymous, symmetric). In terms of the preference relation it may be written as the following axiom

$$
\left(y_{\tau(1)}, y_{\tau(2)}, \ldots, y_{\tau(m)}\right) \cong\left(y_{1}, y_{2}, \ldots, y_{m}\right)
$$

for any permutation $\tau$ of $I$. The preference relations satisfying axioms (4)-(7) we will refer hereafter as impartial rational preference relations.

Further, according to the theory of equity measurement [10], the preference model should satisfy the (Pigou-Dalton) principle of transfers. The principle of transfers states that a transfer of small amount from an outcome to any relatively worse-off outcome results in a more preferred achievement vector. As property of the preference relation, the principle of transfers takes form of the following axiom

$$
y_{i^{\prime}}>y_{i^{\prime \prime}} \Rightarrow \mathbf{y}-\varepsilon \mathbf{e}_{i^{\prime}}+\varepsilon \mathbf{e}_{i^{\prime \prime}} \prec \mathbf{y} \quad \text { for } \quad 0<\varepsilon<y_{i^{\prime}}-y_{i^{\prime \prime}} \text {. }
$$

The preference relations satisfying all axioms (4)-(8) we will call hereafter equitable rational preference relations.

A requirement of impartiality (7) and the principle of transfers (8) complement the multiple criteria optimization axioms (4-6). Therefore, we can consider equitable multiple criteria optimization based on the preference model defined by axioms (4-8). In this paper we develop the basic theory of the equitable multiple criteria linear optimization. The paper is organized as follows. In Section 2, the formal definition of the equitable efficiency is given, and some fundamentals are presented. In Section 3, we develop and analyze generation techniques for equitably efficient solutions. Further, in Section 4 the structure of the equitably efficient set of the multiple criteria linear programs are developed and analyzed.

vol. 33, n 3, 1999 


\section{EQUITABLE EFFICIENCY}

Consider the multiple criteria problem (1) with the preference model defined by equitable rational preference relations. The equitable rational preference relations allow us to define the concept of equitably efficient solution [11], similar to the standard efficient (Pareto-optimal) solution defined with the rational preference relations.

DeFINITION 2.1: We say that achievement vector $\mathbf{y}^{\prime} \in Y$ equitably dominates $\mathbf{y}^{\prime \prime} \in Y$, or $\mathbf{y}^{\prime \prime}$ is equitably dominated by $\mathbf{y}^{\prime}$, iff $\mathbf{y}^{\prime} \prec \mathbf{y}^{\prime \prime}$ for all equitable rational preference relations $\preceq$.

DEFINITION 2.2: We say that achievement vector $\mathbf{y} \in A$ is equitably nondominated, iff there does not exist $\mathbf{y}^{\prime} \in A$ such that $\mathbf{y}^{\prime}$ equitably dominates $\mathbf{y}$.

DEFINITION 2.3: We say that feasible solution $\mathbf{x} \in Q$ is an equitably efficient solution of the mutliple criteria problem (1), iff $\mathbf{y}=\mathbf{C x}$ is equitably nondominated.

Similar to the relation of equitable dominance, we can define the relation of equitable indifference (indifference for all equitable rational preference relations) and the relation of equitable weak dominance (weak preference for all equitable rational preference relations). The relations of equitable dominance $\preceq_{e}$, indifference $\preceq_{e}$ and weak dominance $\preceq_{e}$ satisfy conditions (2-3). So, they define the preference relation $\preceq_{e}$. Moreover, relation $\preceq_{e}$ holds properties of reflexivity (4), transitivity (5), strict monotonicity (6), impartiality (7) and satisfies the principle of transfers (8), thus it is an equitable rational preference relation. Relation $\preceq e$ is the most general equitable rational preference relation and each equitable rational preference relation $\preceq$ is consistent with it in the sense that

$$
\mathbf{y}^{\prime} \preceq_{e} \mathbf{y}^{\prime \prime} \Rightarrow \mathbf{y}^{\prime} \preceq \mathbf{y}^{\prime \prime}
$$

In a similar way, one may introduce the relation of symmetric dominance as the preference for all impartial rational preference relations and define the concept of symmetrically efficient solution. This is equivalent to the concept of symmetry defined by comparisons of permutations of individual outcomes in the achievement vectors [12]. The relation of symmetric dominance $\preceq_{s}$ can be expressed as vector inequality on the ordered achievement vectors. This can be mathematically formalized as follows: introduce the ordering 
map $\Theta: R^{m} \rightarrow R^{m}$ such that $\Theta(\mathbf{y})=\left(\theta_{1}(\mathbf{y}), \theta_{2}(\mathbf{y}), \ldots, \theta_{m}(\mathbf{y})\right)$, where $\theta_{1}(\mathbf{y}) \geq \theta_{2}(\mathbf{y}) \geq \ldots \geq \theta_{m}(\mathbf{y})$ and there exists a permutation $\tau$ of set $I$ such that $\theta_{i}(\mathbf{y})=y_{\tau(i)}$ for $i=1,2, \ldots, m$. Note that due to impartiality axiom (7), $\Theta\left(\mathbf{y}^{\prime}\right)=\Theta\left(\mathbf{y}^{\prime \prime}\right)$ implies $\mathbf{y}^{\prime} \stackrel{\cong}{s}_{s} \mathbf{y}^{\prime \prime}$. In fact [12],

$$
\mathbf{y}^{\prime} \preceq_{s} \mathbf{y}^{\prime \prime} \Leftrightarrow \Theta\left(\mathbf{y}^{\prime}\right) \leqq \Theta\left(\mathbf{y}^{\prime \prime}\right) .
$$

Observe that the relation of equitable dominance $\preceq_{e}$ can be expressed as a vector inequality on the cumulative ordered achievement vectors. Apply to ordered achievement vectors $\Theta(\mathbf{y})$, a linear cumulative map $\Gamma=\left(\gamma_{1}, \gamma_{2}, \ldots, \gamma_{m}\right)$, where for $\mathbf{q} \in R^{m}$

$$
\gamma_{i}(\mathbf{q})=\sum_{j=1}^{i} q_{j} \quad \text { for } \quad i=1,2, \ldots, m .
$$

Composition of these two maps results in the cumulative ordering map $\bar{\Theta}=\left(\bar{\theta}_{1}, \bar{\theta}_{2}, \ldots, \bar{\theta}_{m}\right)$ defined as $\bar{\Theta}(\mathbf{y})=\Gamma(\Theta(\mathbf{y}))$, i.e.,

$$
\bar{\theta}_{i}(\mathbf{y})=\sum_{j=1}^{i} \theta_{j}(\mathbf{y}) \quad \text { for } \quad i=1,2, \ldots, m .
$$

The coefficients of vector $\bar{\Theta}(\mathbf{y})$ express, respectively: the largest outcome, the total of the two largest outcomes, the total of the three largest outcomes, etc.

Directly from the definition of the map $\bar{\Theta}$, it follows that for any two achievement vectors $\mathbf{y}^{\prime}, \mathbf{y}^{\prime \prime} \in Y$ the following relations hold

$$
\begin{gathered}
\Theta\left(\mathbf{y}^{\prime}\right)=\Theta\left(\mathbf{y}^{\prime \prime}\right) \Leftrightarrow \bar{\Theta}\left(\mathbf{y}^{\prime}\right)=\bar{\Theta}\left(\mathbf{y}^{\prime \prime}\right) \\
\Theta\left(\mathbf{y}^{\prime}\right) \leq \Theta\left(\mathbf{y}^{\prime \prime}\right) \Rightarrow \bar{\Theta}\left(\mathbf{y}^{\prime}\right) \leq \bar{\Theta}\left(\mathbf{y}^{\prime \prime}\right) .
\end{gathered}
$$

The reverse implication to (11) is not valid. For instance, $\bar{\Theta}(2,2,2)=$ $(2,4,6) \leq(3,5,6)=\bar{\Theta}(3,2,1)$ and simultaneously $\Theta(2,2,2) \not \leq$ $\Theta(3,2,1)$.

Note that the relation

$$
\mathbf{y}^{\prime} \preceq \mathbf{y}^{\prime \prime} \Leftrightarrow \bar{\Theta}\left(\mathbf{y}^{\prime}\right) \leqq \bar{\Theta}\left(\mathbf{y}^{\prime \prime}\right)
$$

vol. $33, \mathrm{n}^{\circ} 3,1999$ 
is reflexive, transitive and impartial. Moreover, the following formulas hold

$$
\begin{aligned}
& \bar{\Theta}\left(\mathbf{y}^{\prime}\right) \leq \bar{\Theta}\left(\mathbf{y}^{\prime \prime}\right) \Leftrightarrow\left(\bar{\Theta}\left(\mathbf{y}^{\prime}\right) \leqq \bar{\Theta}\left(\mathbf{y}^{\prime \prime}\right) \text { and } \bar{\Theta}\left(\mathbf{y}^{\prime \prime}\right) \$ \bar{\Theta}\left(\mathbf{y}^{\prime}\right)\right) \\
& \bar{\Theta}\left(\mathbf{y}^{\prime}\right)=\bar{\Theta}\left(\mathbf{y}^{\prime \prime}\right) \Leftrightarrow\left(\bar{\Theta}\left(\mathbf{y}^{\prime}\right) \leqq \bar{\Theta}\left(\mathbf{y}^{\prime \prime}\right) \text { and } \bar{\Theta}\left(\mathbf{y}^{\prime \prime}\right) \leqq \bar{\Theta}\left(\mathbf{y}^{\prime}\right)\right) .
\end{aligned}
$$

Thus the relation (12) is a preference relation with the relations of strict preference and indifference defined, respectively, as follows

$$
\begin{aligned}
\mathbf{y}^{\prime} \prec \mathbf{y}^{\prime \prime} & \Leftrightarrow \bar{\Theta}\left(\mathbf{y}^{\prime}\right) \leq \bar{\Theta}\left(\mathbf{y}^{\prime \prime}\right) \\
\mathbf{y}^{\prime} \cong \mathbf{y}^{\prime \prime} & \Leftrightarrow \bar{\Theta}\left(\mathbf{y}^{\prime}\right)=\bar{\Theta}\left(\mathbf{y}^{\prime \prime}\right) .
\end{aligned}
$$

Furthermore

$$
\bar{\Theta}\left(\mathbf{y}-\varepsilon \mathbf{e}_{i}\right) \leq \bar{\Theta}(\mathbf{y}) \quad \text { for } \quad \varepsilon>0, \quad i=1,2, \ldots, m
$$

and

$$
y_{i^{\prime}}>y_{i^{\prime \prime}} \Rightarrow \bar{\Theta}\left(\mathbf{y}-\varepsilon \mathbf{e}_{i^{\prime}}+\varepsilon \mathbf{e}_{i^{\prime \prime}}\right) \leq \bar{\Theta}(\mathbf{y}) \quad \text { for } \quad 0<\varepsilon<y_{i^{\prime}}-y_{i^{\prime \prime}} .
$$

So, the relation (12) is an equitable rational preference relation.

The relation (12) was extensively analyzed within the theory of majorization [13], where it is called the relation of weak submajorization. The theory of majorization includes the result (cf. [13], 2.C.6, p. 28) which can be expressed in the following form.

Proposition 2.1: $\bar{\Theta}\left(\mathbf{y}^{\prime}\right) \leqq \bar{\Theta}\left(\mathbf{y}^{\prime \prime}\right)$, if and only if, there exists a finite sequence of vectors $\mathbf{y}^{0}=\mathbf{y}^{\prime \prime}, \mathbf{y}^{1}, \ldots, \mathbf{y}^{t}$ such that $\mathbf{y}^{k}=\mathbf{y}^{k-1}-\varepsilon_{k} \mathbf{e}_{i^{\prime}}+\varepsilon_{k} \mathbf{e}_{i^{\prime \prime}}$, $0 \leq \varepsilon_{k} \leq y_{i^{\prime}}^{k-1}-y_{i^{\prime \prime}}^{k-1}$ for $k=1,2, \ldots, t$ and $\mathbf{y}^{\prime} \leqq \mathbf{y}^{t}$.

The following propositions and corollaries use this result to show the equivalence of the equitable dominance and the relation (12).

Proposition 2.2: If $\bar{\Theta}\left(\mathbf{y}^{\prime}\right) \leq \bar{\Theta}\left(\mathbf{y}^{\prime \prime}\right)$, then $\Theta\left(\mathbf{y}^{\prime}\right) \leq \Theta\left(\mathbf{y}^{\prime \prime}\right)$ or there exists a finite sequence of vectors $\mathbf{y}^{0}=\mathbf{y}^{\prime \prime}, \mathbf{y}^{1}, \ldots, \mathbf{y}^{t}$ such that $\mathbf{y}^{k}=$ $\mathbf{y}^{k-1}-\varepsilon_{k} \mathbf{e}_{i^{\prime}}+\varepsilon_{k} \mathbf{e}_{i^{\prime \prime}}, 0<\varepsilon_{k}<y_{i^{\prime}}^{k-1}-y_{i^{\prime \prime}}^{k-1}$ for $k=1,2, \ldots, t$ and $\Theta\left(\mathbf{y}^{\prime}\right) \leqq \Theta\left(\mathbf{y}^{t}\right)$.

Proof: From Proposition 2.1, there exists a finite sequence of vectors $\mathbf{y}^{0}=$ $\mathbf{y}^{\prime \prime}, \mathbf{y}^{1}, \ldots, \mathbf{y}^{t}$ such that $\mathbf{y}^{k}=\mathbf{y}^{k-1}-\varepsilon_{k} \mathbf{e}_{i^{\prime}}+\varepsilon_{k} \mathbf{e}_{i^{\prime \prime}}, 0 \leq \varepsilon_{k} \leq y_{i^{\prime}}^{k-1}-y_{i^{\prime \prime}}^{k-1}$ for $k=1,2, \ldots, t$ and $\mathbf{y}^{\prime} \leqq \mathbf{y}^{t}$. Note that $\mathbf{y}^{k-1}-\varepsilon_{k} \mathbf{e}_{i^{\prime}}+\varepsilon_{k} \mathbf{e}_{i^{\prime \prime}}$ is equal to $\mathbf{y}^{k-1}$ for $\varepsilon_{k}=0$, and it is a permutation of $\mathbf{y}^{k-1}$ (where $y_{i^{\prime}}^{k-1}$ and 
$y_{i^{\prime \prime}}^{k-1}$ are swapped) for $\varepsilon_{k}=y_{i^{\prime}}^{k-1}-y_{i^{\prime \prime}}^{k-1}$. Hence, if for all $k=1, \ldots, t$ either $\varepsilon_{k}=0$ or $\varepsilon_{k}=y_{i^{\prime}}^{k-1}-y_{i^{\prime \prime}}^{k-1}$, then $\Theta\left(\mathbf{y}^{\prime}\right) \leqq \Theta\left(\mathbf{y}^{\prime \prime}\right)$ and, due to (10), $\Theta\left(\mathbf{y}^{\prime}\right) \leqq \Theta\left(\mathbf{y}^{\prime \prime}\right)$. Otherwise, $0<\varepsilon_{k}<y_{i^{\prime}}^{k-1}-y_{i^{\prime \prime}}^{k-1}$ for at least one index $k$. Thus there exists a finite sequence of vectors $\mathbf{y}^{0}=\mathbf{y}^{\prime \prime}, \mathbf{y}^{1}, \ldots, \mathbf{y}^{t}$ such that $\mathbf{y}^{k}=\mathbf{y}^{k-1}-\varepsilon_{k} \mathbf{e}_{i^{\prime}}+\varepsilon_{k} \mathbf{e}_{i^{\prime \prime}}, 0<\varepsilon_{k} \leq y_{i^{\prime}}^{k-1}-y_{i^{\prime \prime}}^{k-1}$ for $k=1,2, \ldots, t$ and $\Theta\left(\mathbf{y}^{\prime}\right) \leqq \Theta\left(\mathbf{y}^{t}\right)$.

Proposition 2.3: For any two achievement vectors $\mathbf{y}^{\prime}, \mathbf{y}^{\prime \prime} \in Y$

$$
\mathbf{y}^{\prime} \preceq_{e} \mathbf{y}^{\prime \prime} \Leftrightarrow \bar{\Theta}\left(\mathbf{y}^{\prime}\right) \leqq \bar{\Theta}\left(\mathbf{y}^{\prime \prime}\right) .
$$

Proof: The relation (12) is an equitable rational preference relation. Thus

$$
\mathbf{y}^{\prime} \preceq_{e} \mathbf{y}^{\prime \prime} \Rightarrow \bar{\Theta}\left(\mathbf{y}^{\prime}\right) \leqq \bar{\Theta}\left(\mathbf{y}^{\prime \prime}\right) .
$$

In order to prove the reverse implication, notice that due to Proposition 2.2, for any equitable rational preference relation $\preceq, \Theta\left(\mathbf{y}^{\prime}\right) \preceq \Theta\left(\mathbf{y}^{\prime \prime}\right)$. Hence, due to impartiality of all equitable rational preference relations, $\mathbf{y}^{\prime} \preceq_{e} \mathbf{y}^{\prime \prime}$.

Corollary 2.1: Achievement vector $\mathbf{y}^{\prime} \in Y$ equitably dominates $\mathbf{y}^{\prime \prime} \in Y$, iff $\bar{\Theta}\left(\mathbf{y}^{\prime}\right) \leq \bar{\Theta}\left(\mathbf{y}^{\prime \prime}\right)$.

Note that Corollary 2.1 permits one to express equitable efficiency for problem (1) in terms of the standard efficiency for the multiple criteria problem with objectives $\bar{\Theta}(\mathbf{C x})$

$$
\min \{\bar{\Theta}(\mathbf{C x}): \mathbf{x} \in Q\} .
$$

COROLlary 2.2: A feasible solution $\mathrm{x} \in Q$ is an equitably efficient solution of the multiple criteria problem (1), iff it is an efficient solution of the multiple criteria problem (15).

We emphasize to the reader the importance of this result, as it provides the relationship between equitable efficiency and Pareto-optimality. In what follows we will use Corollary 2.2 to derive properties of the equitably efficient set and to introduce generation techniques.

COROLlary 2.3: For any strictly increasing linear function $s: R \rightarrow R$, vector $\mathbf{x}^{0} \in Q$ is an equitably efficient solution of the multiple criteria problem (1), iff it is an equitably efficient solution of the problem

$$
\min \left\{\left(s\left(\mathbf{c}^{1} \mathbf{x}\right), s\left(\mathbf{c}^{2} \mathbf{x}\right), \ldots, s\left(\mathbf{c}^{m} \mathbf{x}\right)\right): \mathbf{x} \in Q\right\} .
$$


Note that, according to Corollary 2.3, equitable efficiency is scale invariant with respect to linear scaling with a positive factor. This result is very important as such scale invariance is usually considered an axiom of equity measurement.

The equitable dominance relation may be illustrated with the so-called domination structure [14], i.e. a point-to-set map

$$
D(\mathbf{y})=\{\mathbf{d} \in Y: \mathbf{y} \prec \mathbf{y}+\mathbf{d}\} \cup\{\mathbf{0}\} .
$$

For the standard rational dominance relation, the sets $D(\mathbf{y})$ are independent of $\mathbf{y}$ and they take the form of the nonnegative orthant. The domination structure of the equitable dominance depends on the location of an achievement vector $\mathbf{y}$ relative to the absolute equity line $\left(y_{1}=y_{2}=\ldots=\right.$ $\left.y_{m}\right)$. In the general case, the set $D(\mathbf{y})$ is not a cone and it is not convex. Figure 1 shows $D(\mathbf{y})$ fixed at $\mathbf{y}$, i.e. the set $\mathbf{y}+D(\mathbf{y})$.

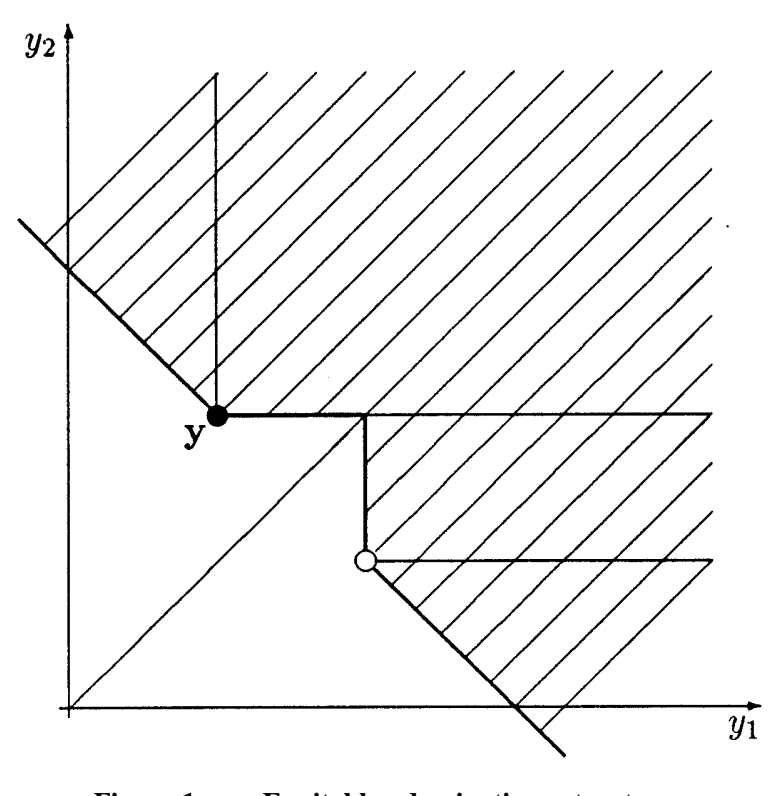

Figure 1. - Equitable domination structure.

Example 2.1: Let us consider a bicriteria problem

$\min \left\{\left(x_{1}, x_{2}\right): 3 x_{1}+x_{2} \geq 21,4 x_{1}+5 x_{2} \geq 72, x_{1} \geq 0, x_{2} \geq 0\right\}$ 


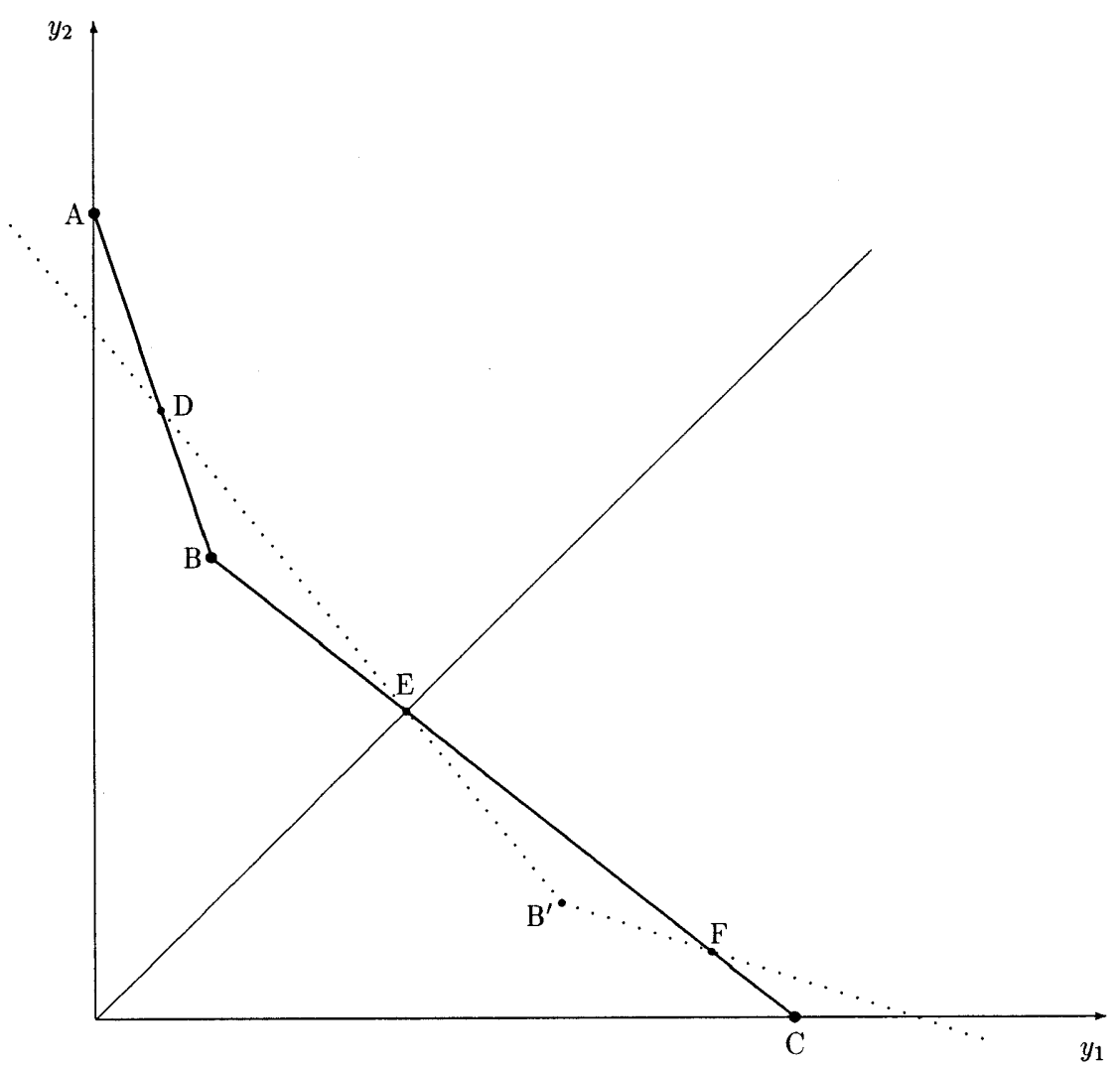

Figure 2. - Graphical illustration for Example 2.1.

Figure 2 gives the graphical illustration of the problem in the objective space $\left(y_{1}, y_{2}\right)$. However, due to the identity objective functions $y_{1}=x_{1}$ and $y_{2}=x_{2}$, it can be directly interpreted in the decision space $\left(x_{1}, x_{2}\right)$. The standard efficient set consists of two line segments $\overline{A B}$ and $\overline{B C}$, where $A=(0,21), B=(3,12)$ and $C=(18,0)$. While taking into account symmetric dominance we eliminate segments $\overline{A D}$ and $\overline{E F}$, where $D=(12 / 7,111 / 7), E=(8,8)$ and $F=(111 / 7,12 / 7)$. Thus, the symmetric efficient set consists of three segments: $\overline{D B}, \overline{B E}$ and $\overline{F C}$. Note that the symmetric efficient set is not connected. Further, taking into account the equitable dominance we get the segment $\overline{B E}$ as the equitably efficient set. In Section 4, we will prove that the equitably efficient set is always connected. 


\section{GENERATION TECHNIQUES}

Efficient solutions of the multiple criteria problem (1) can be generated with simple scalarizations of the problem. Most of them are based on the sum of individual outcomes

$$
\min \left\{\sum_{i=1}^{m} \mathbf{c}^{i} \mathbf{x}: \mathbf{x} \in Q\right\}
$$

or on the minimax approach

$$
\min \left\{\max _{i=1, \ldots, m} \mathbf{c}^{i} \mathbf{x}: \mathbf{x} \in Q\right\}
$$

Scalarization (17) generates efficient solutions as the corresponding preference relation is a rational preference relation. It maintains the properties of reflexivity, transitivity and strict monotonicity. It does not satisfy, however, the principle of transfers. Therefore, scalarization (17), in the general case, may generate solutions which are not equitably efficient. To generate equitably efficient solutions, some convexification is required.

PROPOSITION 3.1: For any strictly convex, increasing function $s: R \rightarrow R$, the preference relation corresponding to the problem

$$
\min \left\{\sum_{i=1}^{m} s\left(\mathbf{c}^{i} \mathbf{x}\right): \mathbf{x} \in Q\right\}
$$

is reflexive, transitive, strictly monotonic, impartial and satisfies the principle of transfers.

Proof: Reflexivity, transitivity, impartiality of the preference relation corresponding to (19) is obvious. Strict monotonicity is due to the strictly increasing function $s$. Thus we only need to prove that the principle of transfers is satisfied. Let $\mathbf{y} \in Y$ and $y_{i^{\prime}}>y_{i^{\prime \prime}}$. Define vectors $\mathbf{y}^{\varepsilon}=\mathbf{y}-\varepsilon \mathbf{e}_{i^{\prime}}+\varepsilon \mathbf{e}_{i^{\prime \prime}}$ and $\mathbf{y}^{s}=\mathbf{y}-\left(y_{i^{\prime}}-y_{i^{\prime \prime}}\right) \mathbf{e}_{i^{\prime}}+\left(y_{i^{\prime}}-y_{i^{\prime \prime}}\right) \mathbf{e}_{i^{\prime \prime}}$. Note that $\mathbf{y}^{\varepsilon}=\lambda \mathbf{y}^{s}+(1-\lambda) \mathbf{y}$, where $\lambda=\varepsilon /\left(y_{i^{\prime}}-y_{i^{\prime \prime}}\right)$, i.e. $0<\lambda<1$ for $0<\varepsilon<y_{i^{\prime}}-y_{i^{\prime \prime}}$. The function $G(\mathbf{y})=\sum_{i=1}^{m} s\left(y_{i}\right)$ is strictly convex and symmetric. Hence

$$
G\left(\mathbf{y}^{\varepsilon}\right)<\lambda G\left(\mathbf{y}^{s}\right)+(1-\lambda) G(\mathbf{y})=G(\mathbf{y}) \quad \text { for } \quad 0<\varepsilon<y_{i^{\prime}}-y_{i^{\prime \prime}}
$$


Thus, the preference relation corresponding to the minimization of $G(\mathbf{y})$ satisfies (8) which completes the proof.

COROLlaRy 3.1: For any strictly convex, increasing function $s: R \rightarrow R$, the optimal solution of the problem (19) is an equitably efficient solution of the multiple criteria problem (1).

Problem (19) corresponds to the expected utility approach. For this study it is not very interesting as it introduces nonlinearity into the multiple criteria linear problem.

Note that Corollary 2.2 allows one to generate equitably efficient solutions of (1) as efficient solutions of problem (15). Scalarization (17), minimizing the sum of outcomes, corresponds to minimization of the last ( $m$-th) objective in problem (15). Similar, the minimax scalarization (18) corresponds to minimization of the first objective in (15). Thus, in the case of bicriteria problems $(m=2)$, the set of equitably efficient solutions is equal to the set of efficient solutions of the bicriteria problem with objectives defined as the maximum and the sum of the original two objectives. In general the following corollary is valid.

COROLlARY 3.2: The set of efficient solutions to the bicriteria problem

$$
\min \left\{\left(\max _{i=1, \ldots, m} \mathbf{c}^{i} \mathbf{x}, \sum_{i=1}^{m} \mathbf{c}^{i} \mathbf{x}\right): \mathbf{x} \in Q\right\}
$$

contains an equitably efficient solution of the multiple criteria problem (1), and if efficient solution $\mathbf{x}^{0}$ of (20) for all $\mathbf{x} \in Q$ satisfies

$$
\begin{gathered}
\max _{i=1, \ldots, m} \mathbf{c}^{i} \mathbf{x}=\max _{i=1, \ldots, m} \mathbf{c}^{i} \mathbf{x}^{0} \\
\text { and } \quad \sum_{i=1}^{m} \mathbf{c}^{i} \mathbf{x}=\sum_{i=1}^{m} \mathbf{c}^{i} \mathbf{x}^{0} \Rightarrow \Theta(\mathbf{C x})=\Theta\left(\mathbf{C x}^{0}\right)
\end{gathered}
$$

then $\mathrm{x}^{0}$ is an equitably efficient solution of (1).

In the case of efficiency one may use the scalarization (17) with weighted objective functions to generate various efficient solutions. In fact, it provides a complete parametrization of the efficient set for multiple criteria linear programs. In the case of equitable multiple criteria programming one cannot assign various weights to individual objective functions, as that violates the requirement of impartiality (7). However, due to Corollary 2.2,

vol. $33, \mathrm{n}^{\circ} 3,1999$ 
tthe weighting approach can be applied to problem (15) resulting in the scalarization

$$
\min \left\{\sum_{i=1}^{m} w_{i} \bar{\theta}_{i}(\mathbf{C x}): \mathbf{x} \in Q\right\}
$$

note that, due to the definition of map $\bar{\Theta}$ with (9), the above problem can be expressed in the form with weights $\bar{w}_{i}=\sum_{j=i}^{m} w_{j}(i=1,2, \ldots, m)$ allocated to coefficients of the ordered achievement vector. Such an approach to multiple criteria optimization was introduced [15] as the so-called Ordered Weighted Averaging (OWA). When applying OWA to problem (1) we get

$$
\min \left\{\sum_{i=1}^{m} w_{i} \theta_{i}(\mathbf{C x}): \mathbf{x} \in Q\right\} .
$$

PROPOSITION 3.2: If weights $w_{i}$ are strictly decreasing and positive, i.e.

$$
w_{1}>w_{2}>\ldots>w_{m-1}>w_{m}>0
$$

then each optimal solution of the OWA problem (22) is an equitably efficient solution of (1).

Proof: Problem (22) with weights $w_{i}$ can be expressed in the form

$$
\min \left\{\sum_{i=1}^{m} w_{i}^{\prime} \bar{\theta}_{i}(\mathbf{C x}): \mathbf{x} \in Q\right\}
$$

where coefficients $w_{i}^{\prime}$ are defined as $w_{m}^{\prime}=w_{m}$ and $w_{i}^{\prime}=w_{i}-w_{i+1}$ for $i=1,2, \ldots, m-1$.

If (23) holds, then $w_{i}^{\prime}>0$ for $i=1,2, \ldots, m$. Thus, due to Corollary 2.2, each optimal solution of (22) is an equitably efficient solution of (1).

Note that for weights $w_{i}$ satisfying (23), for any permutation $\tau$ of $I$ the following inequality holds

$$
\sum_{i=1}^{m} w_{\tau(i)} y_{i} \leq \sum_{i=1}^{m} w_{i} \theta_{i}(\mathbf{y})
$$

Thus, the OWA problem (22) may be written as the linear program

$\min z$

subject to $\mathbf{x} \in Q$ 


$$
\begin{aligned}
& y_{i}=\mathbf{c}^{i} \mathbf{x} \quad \text { for } \quad i=1,2, \ldots, m \\
& z \geq \sum_{i=1}^{m} w_{\tau(i)} y_{i} \quad \text { for } \quad \tau \in \Pi
\end{aligned}
$$

where $\Pi$ denotes the set of all permutations $\tau$ of $I$.

In problem (24-27) the ordering operator $\Theta$ is replaced with $m$ ! linear inequalities (27). It generates a large number of constraints but all the inequalities (27) are defined by permutations of the single vector of weights $w_{i}$. Therefore, the dual of (24-27) can be solved by the simplex method with the column generation technique applied to columns corresponding to (27).

Applying the lexicographic optimization to problem (15) we get the lexicographic problem

$$
\operatorname{lexmin}\{\bar{\Theta}(\mathbf{C x}): \mathbf{x} \in Q\}
$$

where first $\bar{\theta}_{1}(\mathbf{C x})$ is minimized, next $\bar{\theta}_{2}(\mathbf{C x})$ and so on. Due to (9), problem (28) is equivalent to the problem

$$
\operatorname{lexmin}\{\Theta(\mathbf{C x}): \mathbf{x} \in Q\}
$$

which represents the lexicographic minimax approach to the original multiple criteria problem (1). Problem (29) is a regularization of the standard minimax scalarization (18), but in the former in addition to the largest outcome we minimize also the second largest outcome (provided that the largest one remains as small as possible), minimize the third largest (provided that the two largest remain as small as possible), and so on. The lexicographic minimax solution is known in the game theory as the nucleolus of a matrix game [16]. This approach has been recently used for linear programming problems related to multiperiod resource allocation [7] and for linear multiple criteria problems [17]. The lexicographic minimax problem (29) may be considered a limiting case of the OWA problem (22) when the differences among the weights increase to infinity. As the lexicographic optimization generates efficient solutions, thus due to Corollary 2.2, we get the following corollary.

COROLlary 3.3: The optimal solution of the lexicographic minimax problem (29) is an equitably efficient solution of the multiple criteria problem (1).

The lexicographic minimax solution is unique with respect to the ordered achievement vectors $\Theta(\mathbf{C x})$. It can be considered in some sense the "most 
equitable solution". Note that one may wish to consider the problem (15) as an equitable problem (with an equitable rational preference relation). In such a situation we should apply Corollary 2.2 to problem (15). It results in the problem with doubly cumulative ordered criteria which again may be considered as equitable. As the limit of such an approach we get the lexicographic minimax problem (29). One may wish to look for the "least equitable solution" applying reverse lexicographic minimization to the problem (15), i.e. solving the lexicographic problem

$$
\operatorname{lexmin}\left\{\left(\bar{\theta}_{m}(\mathbf{C x}), \bar{\theta}_{m-1}(\mathbf{C x}), \ldots, \bar{\theta}_{1}(\mathbf{C x})\right): \mathbf{x} \in Q\right\}
$$

where first $\bar{\theta}_{m}(\mathbf{C x})$ is minimized, next $\bar{\theta}_{m-1}(\mathbf{C x})$ and so on. While the lexicographic minimax (29) is a refinement of the standard minimax approach (18), the problem (30) is a lexicographic refinement of the scalarization (17). Note, that in the lexicographic optimization problem dividing objectives by constants does not affect the solution and $\bar{\theta}_{i}(\mathbf{y}) / i$ represents the mean of $i$ largest coefficients in the achievement vector $\mathbf{y}$. Therefore, we refer to problem (30) as the lexicographic mean problem. As the lexicographic optimization generates efficient solutions, from Corollary 2.2, we get the following corollary.

Corollary 3.4: The optimal solution of the lexicographic mean problem (30) is an equitably efficient solution of the multiple criteria problem (1).

\section{STRUCTURE OF EQUITABLY EFFICIENT SET}

In Section 2 we established the equivalence between the equitably efficient solutions of (1) and the efficient solutions of problem (15) (compare Cor. 2.2). Now, we further use this result to explore the structure of the equitably efficient set. Note that the individual objective functions of problem (15) are convex piecewise linear functions of $\mathbf{y}$. They can be written in the form

$$
\bar{\theta}_{i}(\mathbf{y})=\max _{\tau \in \Pi}\left(\sum_{k=1}^{i} y_{\tau(k)}\right)
$$

where $\Pi$ denotes the set of all permutations $\tau$ of the index set $I$. Thus, the corresponding problem (15) can be expressed in the form of multiple criteria linear program

$$
\min \left(z_{1}, z_{2}, \ldots, z_{m}\right)
$$




$$
\begin{aligned}
& \text { subject to } \\
& \mathbf{x} \in Q \\
& y_{i}=\mathbf{c}^{i} \mathbf{x} \quad \text { for } \quad i=1,2, \ldots, m \\
& z_{i} \geq \sum_{k=1}^{i} y_{\tau(k)} \quad \text { for } \quad \tau \in \Pi ; i=1,2, \ldots, m .
\end{aligned}
$$

Due to (31), multiple criteria linear program (32-35) is equivalent to problem (15) as stated in the following proposition.

Proposition 4.1: A triple $\left(\mathbf{x}^{0}, \mathbf{y}^{0}, \mathbf{z}^{0}\right)$ is an efficient solution of (32-35), if and only if, $\mathbf{y}^{0}=\mathbf{C x}^{0}, \mathbf{z}^{0}=\bar{\Theta}\left(\mathbf{y}^{0}\right)$ and $\mathbf{x}^{0}$ is an efficient solution of problem (15).

In considering the equitably efficient set, there are some basic facts which should be introduced. From the following result, we can appreciate that the new concept is consistent with Pareto optimization, but is a refinement. The motivation for making such a refinement derives from the application setting, in general. However, due to the nature of Pareto optimization, it is also often desirable to narrow the solution set and considering equitably efficient points is a natural way to select solutions.

Proposition 4.2: Any equitably efficient solution is an efficient solution.

Proof: Let $\mathbf{x}^{0}$ be an equitable efficient solution. Suppose that $\mathbf{x}^{0}$ is not efficient in the Pareto-optimal sense. Then a feasible vector $\mathbf{x}$ must exist such that the achievement vectors $\mathbf{y}^{0}=\mathbf{C x}^{0}$ and $\mathbf{y}=\mathbf{C x}$ satisfy $\mathbf{y} \leq \mathbf{y}^{0}$. Thus $\Theta(\mathbf{y}) \leq \Theta\left(\mathbf{y}^{0}\right)$ and $\bar{\Theta}(\mathbf{y}) \leq \bar{\Theta}\left(\mathbf{y}^{0}\right)$. Hence, $\mathbf{y}$ equitably dominates $\mathbf{y}^{0}$ (Cor. 2.1), which contradicts the equitable efficiency of $\mathbf{x}^{0}$.

As observed earlier, the domination structure (16) related to equitable efficiency is not necessarily a cone and may not be a convex set (see Fig. 1). Thus questions of existence of equitably efficient solutions arise. The next results show that some naturally occurring sufficient conditions resolve this tissue.

Proposition 4.3: If $Q \neq \emptyset$ and there exists $\mathbf{y}^{*} \in Y$ such that $\mathbf{y}^{*} \preceq_{e} \mathbf{C x}$ for all $\mathbf{x} \in Q$, then there exists an equitably efficient solution of problem (1).

Proof: Due to Proposition 2.3, $\mathbf{z}^{*}=\bar{\Theta}\left(\mathbf{y}^{*}\right) \leqq \bar{\Theta}(\mathbf{C x})$ for all $\mathbf{x} \in Q$. Thus, $z^{*} \leqq \mathbf{z}$ for any attainable achievement vector $\mathbf{z}$ of the multiple criteria linear program (32-35), which is by Proposition 4.1 equivalent to the problem (15). Hence, there exists an efficient solution $\mathbf{x}^{0}$ of (32)-(35) [1]. Due to Corollary $2.2, \mathrm{x}^{0}$ is an equitably efficient solution of problem (1). 
Proposition 4.4: If there exists an efficient solution of problem (1), then there exists an equitably efficient solution of problem (1).

Proof: If $\mathbf{x}^{0} \in Q$ is an efficient solution of problem (1), then there exist positive weights $w_{i}(i=1,2, \ldots, m)$ such that

$$
\sum_{i=1}^{m} w_{i} \mathbf{c}^{i} \mathbf{x}^{0} \leq \sum_{i=1}^{m} w_{i} \mathbf{c}^{i} \mathbf{x} \quad \text { for } \quad \mathbf{x} \in Q .
$$

Hence, in the criterion space

$$
\sum_{i=1}^{m} w_{i} y_{i} \geq w_{0} \quad \text { for } \quad \mathbf{y} \in A
$$

where $w_{0}=\sum_{i=1}^{m} w_{i} \mathbf{c}^{i} \mathbf{x}^{0}$. From (36), it follows that

$$
\max _{i=1, \ldots, m} y_{i} \geq w_{0} /\left(\sum_{i=1}^{m} w_{i}\right) \quad \text { for } \quad \mathbf{y} \in A .
$$

Hence, there exists an optimal solution of the minimax problem

$$
\min \left\{\max _{i=1, \ldots, m} y_{i}: \mathbf{y} \in A\right\} \text {. }
$$

Moreover, due to (36), each optimal solution $\overline{\mathbf{y}}$ of problem (37) must satisfy inequalities

$$
\bar{y}_{i} \geq\left(w_{0}-\left(\bar{w}-w_{i}\right) z^{*}\right) / w_{i} \quad \text { for } \quad i=1,2, \ldots, m
$$

where $\bar{w}=\sum_{i=1}^{m} w_{i}$, and $z^{*}$ denotes the optimal value of the minimax problem (37). Thus, there exists the optimal solution of the lexicographic minimax problem (29) which, due to Corollary 3.3, is an equitably efficient solution of the multiple criteria problem (1).

The existence of the equitably efficient set and its relationship to the efficient set have been discussed. However, now we point out the fact that the equitably efficient set does not suffer from the difficulties of the symmetric efficient set (lack of connectivity, see Ex. 2.1) and it is therefore a more useful and convenient solution set, both from the theoretical and the practical points of view.

Proposition 4.5: The equitably efficient set of problem (1) is connected.

Proof: The equitably efficient set of problem (1) is the same as the efficient set of problem (15). From Proposition 4.1, the efficient solutions of 
problem (15) are in one-to-one correspondence to the efficient solutions of (32-35). Since (32-35) is a linear multiple criteria optimization problem, its efficient set is a connected set [18].

A very important result in multiple objective linear optimization is the association of efficient solutions with optimal solutions of the scalar weighting problem using positive weights [1]. The following result is the analogue for our equitably efficient solution set. It seems that such a result should thus play an important role in analysis of equitable efficiency.

Proposition 4.6: A feasible solution $\mathbf{x}^{0} \in Q$ is equitably efficient, if and only if, there exist strictly decreasing and positive weights $w_{i}$ (i.e. weights satisfying (23)) such that $\mathbf{x}^{0}$ is an optimal solution of the corresponding OWA problem (22).

Proof: Sufficiency of the condition follows from Proposition 3.2. Thus we only need to show that for each equitably efficient solution $\mathbf{x}^{0} \in Q$ there exist strictly decreasing and positive weights $w_{i}$ (i.e. weights satisfying (23)) such that $\mathbf{x}^{0}$ is an optimal solution of the corresponding OWA problem (22).

Due to Proposition 4.1, if $\mathrm{x}^{0}$ is an equitably efficient solution of (1), then $\left(\mathbf{x}^{0}, \mathbf{C x}^{0}, \bar{\Theta}\left(\mathbf{C x}^{0}\right)\right)$ is an efficient solution of multiple criteria linear program (32-35). Thus, from the theory of multiple criteria linear optimization [1], there exist positive weights $\bar{w}_{i}(i=1,2, \ldots, m)$ such that $\left(\mathbf{x}^{0}, \mathbf{C x}^{0}, \bar{\Theta}\left(\mathbf{C x}^{0}\right)\right)$ is an optimal solution of the problem

$$
\min \left\{\sum_{i=1}^{m} \bar{w}_{i} z_{i}:(33)-(35)\right\} \text {. }
$$

Due to positive weights $\bar{\omega}_{i}$, the above problem is equivalent to the problem

$$
\min \left\{\sum_{i=1}^{m} \bar{w}_{i} \bar{\theta}_{i}(\mathbf{C x}): \mathbf{x} \in Q\right\}
$$

which, by definition of the map $\bar{\Theta}$ with (9), can be expressed as the OWA problem (22) weights $w_{i}=\sum_{j=i}^{m} \bar{w}_{j}(i=1,2, \ldots, m)$. Moreover, weights $w_{i}$ satisfy the requirement (23). Thus, there exist strictly decreasing and positive weights $w_{i}$ such that $\mathbf{x}^{0}$ is an optimal solution of the corresponding OWA problem (22).

Recall that in Section 3 several generation techniques for obtaining equitably efficient solutions were derived. Since the equitably efficient set is

vol. $33, \mathrm{n}^{\circ} 3,1999$ 
a connected subset of the efficient set, it is of interest to derive additional results which could potentially provide an easy way to enter to enter the equitably efficient set as well as serve as an aid to locating the equitably efficient solutions within the efficient set.

PROPOSITION 4.7: If there exists any efficient solution $\mathrm{x}^{0}$ of (1) with equal outcomes $\mathbf{c}^{1} \mathbf{x}^{0}=\mathbf{c}^{2} \mathbf{x}^{0}=\ldots=\mathbf{c}^{m} \mathbf{x}^{0}$, then it is an equitably efficient solution.

Proof: An efficient solution with equal outcomes is a lexicographic minimax solution. Hence, by Corollary 3.3 , such a solution is equitably efficient.

Proposition 4.8: A feasible solution $\mathbf{x}^{0} \in Q$ such that for some permutation $\tau$

$$
\mathbf{c}^{\tau(1)} \mathbf{x}^{0}>\mathbf{c}^{\tau(2)} \mathbf{x}^{0}>\ldots>\mathbf{c}^{\tau(m)} \mathbf{x}^{0}
$$

is equitably efficient, if and only if, there exist strictly decreasing and positive weights $w_{i}$ (i.e. weights satisfying (23)) such that $\mathbf{x}^{0}$ is an optimal solution of the linear problem

$$
\min \left\{\sum_{i=1}^{m} w_{i} \mathbf{c}^{\tau(i)} \mathbf{x}: \mathbf{x} \in Q\right\}
$$

Proof: If for $\mathbf{x}^{0} \in Q$ satisfying (38) there exist strictly decreasing and positive weights $w_{i}$ such that $\mathbf{x}^{0}$ is an optimal solution of the linear problem (39), then

$$
\sum_{i=1}^{m} w_{i} \theta_{i}\left(\mathbf{C x}^{0}\right)=\sum_{i=1}^{m} w_{i} \mathbf{c}^{\tau(i)} \mathbf{x}^{0} \leq \sum_{i=1}^{m} w_{i} \mathbf{c}^{\tau(i)} \mathbf{x} \leq \sum_{i=1}^{m} w_{i} \theta_{i}(\mathbf{C x})
$$

for each $\mathrm{x} \in Q$. Thus due to Proposition 4.6, $\mathrm{x}^{0}$ is an equitably efficient solution of (1).

From Proposition 4.6, there exist strictly decreasing and positive weights $w_{i}$ (i.e. weights satisfying (23)) such that $\mathbf{x}^{0}$ is an optimal solution of the corresponding OWA problem (22). We will show that $\mathbf{x}^{0}$ satisfying (38) is also an optimal soluton of the corresponding problem (39) with the same weights. If not, then there exists $\mathbf{x}^{1} \in Q$ such that $\sum_{i=1}^{m} w_{i} \mathbf{c}^{\tau(i)} \mathbf{x}^{1}<$ $\sum_{i=1}^{m} w_{i} \mathbf{c}^{\tau(i)} \mathbf{x}^{0}$. Note that due to convexity of the feasible set $Q$, for any $0<\varepsilon<1$ vector $\mathbf{x}^{\varepsilon}=(1-\varepsilon) \mathbf{x}^{0}+\varepsilon \mathbf{x}^{1}$ is a feasible solution and 
$\sum_{i=1}^{m} w_{i} \mathbf{c}^{\tau(i)} \mathbf{x}^{\varepsilon}<\sum_{i=1}^{m} w_{i} \mathbf{c}^{\tau(i)} \mathbf{x}^{0}$. Moreover, there exists $\varepsilon_{0}>0$ such that for all $0<\varepsilon<\varepsilon_{0}$

$$
\mathbf{c}^{\tau(1)} \mathbf{x}^{\varepsilon}>\mathbf{c}^{\tau(2)} \mathbf{x}^{\varepsilon}>\ldots>\mathbf{c}^{\tau(m)} \mathbf{x}^{\varepsilon} .
$$

Hence, for sufficiently small positive $\varepsilon$

$$
\sum_{i=1}^{m} w_{i} \theta_{i}\left(\mathbf{C} \mathbf{x}^{\varepsilon}\right)=\sum_{i=1}^{m} w_{i} \mathbf{c}^{\tau(i)} \mathbf{x}^{\varepsilon}<\sum_{i=1}^{m} w_{i} \mathbf{c}^{\tau(i)} \mathbf{x}^{0}=\sum_{i=1}^{m} w_{i} \theta_{i}\left(\mathbf{C x}^{0}\right)
$$

which contradicts optimality of $\mathbf{x}^{0}$ for the OWA problem.

In the case of two-dimensional criterion space $(m=2)$, either $f_{1}(\mathbf{x})$ $\leq f_{2}(\mathbf{x})$ or $f_{1}(\mathbf{x}) \geq f_{2}(\mathbf{x})$ for all equitably efficient solutions of problem (1). That means there exists a permutation $\tau \in \Pi$ such that for each equitably efficient solution $x^{0}$ of problem (1) the following inequality holds

$$
\mathbf{c}^{\tau(1)} \mathbf{x}^{0} \geq \mathbf{c}^{\tau(2)} \mathbf{x}^{0} \geq \ldots \geq \mathbf{c}^{\tau(m)} \mathbf{x}^{0} .
$$

This property is not valid for an arbitrary number of criteria. We illustrate this the following example.

Example 4.1: Let us consider a simple problem with four criteria

$$
\begin{gathered}
\min \left\{\left(10 x_{1}+12 x_{2}, 8 x_{1}+6 x_{2}, 6 x_{1}+8 x_{2}, 4 x_{1}\right): x_{1}+x_{2}=1,\right. \\
\left.x_{1} \geq 0, x_{2} \geq 0\right\}
\end{gathered}
$$

The attainable set for this problem is the line segment connecting two vortices: $\mathbf{y}^{\prime}=(10,8,6,4)$ and $\mathbf{y}^{\prime \prime}=(12,6,8,0)$. Note that $y_{2}^{\prime}>y_{3}^{\prime}$ whereas $y_{2}^{\prime \prime}<y_{3}^{\prime \prime}$. Nevertheless, both $\mathbf{y}^{\prime}$ and $\mathbf{y}^{\prime \prime}$ are equitably nondominated. In fact, all the attainable achievement vectors are equitably nondominated.

\section{CONCLUSIONS}

In this paper a theoretical development of a new concept of solution of a linear mutliple criteria optimization problem is introduced. From a foundation of rational preference relations and several additional axioms one obtains the concept of equitable efficiency. By utilizing the results concerning the ordering of achievement vectors and several related ideas, it is possible 
to obtain a linear multiple criteria optimization problem which serves in a surrogate role. That is, by seeking efficient solutions of this new problem, we find equitably efficient solutions of the original problem. Utilizing this key result, some properties of the equitably efficient set are obtained. These include sufficient conditions for existence, connectivity of the equitably efficient set, and characterizations related to weighting problems. Some illustrative examples point to advantages of equitably efficient solutions, as well as to richness relative to their variety.

We do not suggest equitable efficiency as a replacement for Paretooptimality. On the contrary, it seems to be a supplemental concept, which is rather highly dependent on the existing theory and thus it is actually quite accessible to those using the Pareto theory. There are several multiple criteria decision problems where the Pareto-optimal solution concept is not powerful enough and the equity of individual outcomes is an important issue. Uniform and equitable outcomes arise in many practical problems. We do suggest that the concept of equitable efficiency allows for a rigorous solution of such problems which are not handled in a satifactory way by general Pareto theory.

\section{REFERENCES}

1. R. E. STEUER, Multiple Criteria Optimization - Theory, Computation and Applications, John Wiley and Sons, New York, 1986.

2. T. J. Stewart, A Critical Survey on the Status of Multiple Criteria Decision Making Theory and Practice, OMEGA, 1992, 20, p. 569-586.

3. D. J. White, A Bibliography on the Applications of Mathematical Programming Multiple-Objective Methods, J. Oper. Res. Soc., 1990, 41, p. 669-691.

4. T. IвaRaki and N. Катон, Resource Allocation Problems, Algorithmic Approaches, MIT Press, Cambridge, Massachusetts, 1988.

5. J. R. Brown, The Sharing Problem, Oper. Res., 1979, 27, p. 324-340.

6. J. R. BRown, The Knapsack Sharing Problem, Oper. Res., 1979, 27, p. 341-355.

7. R. S. KLein, H. Luss and D. R. Smith, A Lexicographic Minimax Algorithm for Multiperiod Resource Allocation, Math. Programming, 1992, 55, p. 213-234.

8. W. Ogryczak, On the Lexicographic Minimax Approach to Location Problems, European J. Oper. Res., 1997, 100, p. 566-595.

9. Ph. Vincke, Multicriteria Decision-Aid, John Wiley and Sons, New York, 1992.

10. A. SEN, On Economic Inequality, Clarendon Press, Oxford, 1973.

11. W. OGryczak, Equitable Multiple Criteria Programming, Warsaw University, Institute of Informatics, Technical Report TR 96-02 (223), 1996.

12. V. V. PodinovskiI, Multi-Criterion Problems with Uniform Equivalent Criteria, U.S.S.R. Comput. Math. and Math. Phys., 1975, 15, p. 47-60.

13. A. W. Marshall and I. Olkin, Inequalities: Theory of Majorization and Its Applications, Academic Press New York, New York, 1979. 
14. P. L. Yu, Cone Convexity, Cone Extreme Points, and Nondominated Solutions in Decisions Problems with Multiple Objectives, J. Optim. Theor. Appl., 1974, 14, p. 319-377.

15. R. R. YAGER, On Ordered Weighted Averaging Aggregation Operators in Multicriteria Decision Making, IEEE Trans. Systems Man Cybern., 1988, 18, p. 183-190.

16. J. A. M. Potters and S. H. Tus, The Nucleolus of a Matrix Game and Other Nucleoli, Math. Oper. Res., 1992, 17, p. 164-174.

17. E. Marchi and J. A. Oviedo, Lexicographic Optimality in the Multiple Objective Linear Programming: the Nucleolar Solution, Eur. J. Oper. Res., 1992, 57, p. 355359.

18. D. T. Luc, Theory of Vector Optimization, Springer-Verlag, Berlin, 1989. 